Recepción: 18 / 05 / 2018

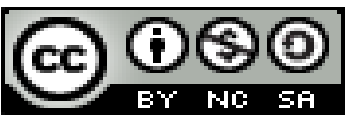

Aceptación: 27 / 08 / 2018

Publicación: 05 / 10 / 2018

\title{
Uso de las tecnológicas de la información y comunicación (tic) en los institutos de educación superior
}
Use of information and communication technologies (tic) in higher education institutes

\section{Uso de tecnologias de informação e comunicação (tic) em institutos de ensino superior}

\author{
Javier Guaña-Moya ${ }^{\text {I }}$ \\ eguana953@puce.edu.ec \\ Norma Luna-Echeverría II \\ nluna@itscompusur.edu.ec \\ Andrés Erazo-Luna III \\ aerazo@itscompusur.edu.ec \\ Fredy Huebla-Huebla IV \\ fhuebla@itscompusur.edu.ec
}

Correspondencia: eguana953@puce.edu.ec

\footnotetext{
${ }^{\mathrm{I}}$ Doctor en Ciencias Informáticas, Docente de la Pontificia Universidad Católica del Ecuador, Quito, Ecuador.

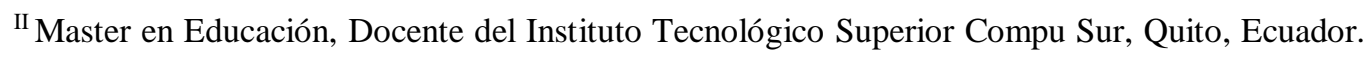

${ }^{\text {III }}$ Master en Educación, Docente del Instituto Tecnológico Superior Compu Sur, Quito, Ecuador.

IV Master en Educación, Docente del Instituto Tecnológico Superior Compu Sur, Quito, Ecuador.
} 


\section{Resumen}

La sociedad en la actualidad, se encuentra en evolución constante en las nuevas tecnologías de la información y comunicación, lo que ha permitido el manejo de la información de una manera más ágil proporcionando así una nueva estructura organizacional, es por ello que los Institutos de Educación Superior, ha incorporado en sus mallas asignaturas de Contabilidad, Administración, Informática, etc., y de acuerdo al avance de la tecnología y la mejora en los procesos de educación presencial, semipresencial y virtual, se ha visto en la necesidad de la utilización de nuevas aplicaciones informáticas, que facilite la enseñanza y aprendizaje entre los docentes y estudiantes para lograr un mejor desarrollo en las actividades docentes y la comprensión de los estudiantes, de manera óptima, logrando así la inserción de estos nuevos procesos en el campo profesional y personal. Es importante recalcar que, en esta investigación se puede especificar que de acuerdo al análisis de datos, más del $67 \%$ de los docentes siguen trabajando con clases magistrales y sin apoyo de las TIC, por lo que más del $60 \%$ de profesores encuestados describen que se necesita las aplicaciones tecnológicas pero no se ha aplicado en clase, se puede describir también que las Tecnologías de la Información y Comunicación (TIC) han permitido desarrollar nuevas habilidades, estrategias y procesos de enseñanza aprendizaje, logrando un $60 \%$ más de concentración en los estudiantes universitarios en las diferentes asignaturas.

Palabras clave: TIC; educación; tecnología, EVEA.

\section{Abstract}

Society today is constantly evolving in the new information and communication technologies, which has allowed the handling of information in a more agile way, thus providing a new organizational structure, that is why the Institutes of Education Superior, has incorporated accounting, administrative, computing, etc. subjects into its meshes, and according to the advancement of technology and the improvement in face-to-face, blended and virtual education processes, it has been necessary to use new computer applications, which facilitate teaching and learning among teachers and students to achieve better development in teaching activities and student understanding, optimally, thus achieving the insertion of these new processes in the professional and personal field. It is important to emphasize that, in this research, it can be specified that according to the teachers' answers, more than $67 \%$ of the teachers continue to work 
Javier Guaña Moya, Norma Luna Echeverría, Andrés Erazo Luna, Fredy Huebla Huebla

with master classes and without ICT support, so that more than $60 \%$ of the teachers surveyed 
describe that technological applications are needed but has not been applied in class, it can also be described that Information and Communication Technologies (ICT) have allowed to develop new skills, strategies and teaching-learning processes, achieving 60\% more concentration in the university students in the different subjects.

Key words: TIC; education; technology; EVEA.

\section{Resumo}

A sociedade atual está em constante evolução nas novas tecnologias de informação e comunicação, o que tem permitido o manuseio das informações de forma mais ágil, proporcionando uma nova estrutura organizacional, por isso os Institutos de Educação superior, tem incorporada seus sujeitos malhas de contabilidade, Administração, iT, etc, e de acordo com o avanço da tecnologia e processos melhorados cara, educação misturados e virtual, tem sido a necessidade para a utilização de novas aplicações informáticas, que facilitam o ensino e aprendizagem entre professores e alunos para alcançar um melhor desenvolvimento das atividades de ensino e compreensão do aluno, de forma otimizada, conseguindo assim a inserção destes novos processos no campo profissional e pessoal. É importante ressaltar que esta pesquisa pode ser especificado que, segundo a análise de dados, mais de $67 \%$ dos professores continuar a trabalhar com o mestre, sem aulas de apoio das TIC, de modo que mais de $60 \%$ dos professores entrevistados relatório que aplicações tecnológicas são necessárias, mas não foram aplicadas em sala de aula, também pode ser descrito que as Tecnologias de Informação e Comunicação (TIC) permitiram o desenvolvimento de novas habilidades, estratégias e processos de ensinoaprendizagem, alcançando concentração de $60 \%$ a mais. Estudantes universitários nos diferentes assuntos.

Palavras chave: TIC; educação; tecnologia, EVEA.

\section{Introducción}

Los nuevos procesos de enseñanza aprendizaje en sectores comunitarios en el mundo, se basa en procesos constructivos del conocimiento, por ello, se concluye según índices obtenidos por UNESCO 2015, que el crecimiento de la población ha sido a pasos acelerados, por ello es importante que las políticas públicas de los diferentes gobiernos formen parte de la aplicación de 
nuevas estrategias metodológicas de aprendizaje, permitiendo así eliminar el analfabetismo en sus habitantes, y promoviendo nuevas oportunidades de contenido y la generación de nuevos conocimientos, (Segrera, 2015).

Es importante insistir que los procesos educativos han evolucionado de forma inmensurable, logrando un proceso activo de educación y cambiando los paradigmas de enseñanza, logrando así que la educación sea de forma constructivista, (Moya, 2017), por ello, hay que describir que estos nuevos procesos de cambio en la educación, la introducción de las redes sociales al ámbito educativo y la inserción de las Tecnologías de la Información y Comunicación (TIC), han ayudado al mejor desarrollo de los procesos educativos, (Guaña-Moya J. Q.-A.-T., 2018 ).

De acuerdo al informe mundial de la UNESCO, (2005), describe que es importante promover la utilización de nuevas estrategias y métodos educativos innovadores acordes a las circunstancias del país y basados en tecnologías de información que formen personas críticas y capaces de resolver los problemas que van surgiendo en el camino, (Burch, 2005).

Es por ello que, los nuevos avances en la educación han permitido que las comunicaciones en la actualidad se desarrollan en tiempo real, lo que se evidencia con los procedimientos basados en la web, las comunicaciones, la informática, telecomunicaciones y otras tecnologías informáticas existentes en la actualidad, logrando así una evolución en los procesos educativos, basados en la era de la información, así como también en las nuevas plataformas de entornos virtuales de enseñanza aprendizaje (EVEA), las cuales ofrecen más servicios que satisfacen la necesidad en todas los usuarios, (Guaña-Moya E. J.-Q.-R., 2015).

Es importante recalcar que Chavez-Burbano, (2017), estipula que, en las instituciones educativas del mundo, ya se están utilizando la red del correo electrónico, el cual permite la interacción con los docentes y estudiantes, teniendo aplicaciones amigables y de fácil uso, para ello cada día se crean nuevas alternativas que posibiliten un mejor servicio multimedia en una red, ofertando así mejor calidad en el servicio y mayor accesibilidad para que el internet llegue utilizarse a nivel global.

Extiendo una nueva visión educativa, (Valverde Berrocoso, 2014), propone recurrir a la suma etnográfica mundial de las técnicas antiguas cuyo análisis surgió de las relaciones sociales y la experiencia de formación online que se ha presentado por una revolución para la Educación 
Superior, también (Salinas, 2004), afirma que, las instituciones de educación superior deben ser más flexibles y deben desarrollar nuevas vías de integración a través de las TIC en los procesos de formación, logrando así que los estudiantes se adapten a las nuevas necesidades de la sociedad actual.

\section{Metodología}

La presente investigación, se basa en el análisis de resultados del estudio que involucra a docentes y estudiantes de los Institutos de Educación Superior, donde se imparte la asignatura de Contabilidad, Administración, Informática, etc., por ello se trabajó con dos actividades basados en las encuestas dirigidos a 40 docentes que imparten las cátedras en los Institutos Tecnológicos Superiores y como segundo momento encuestas dirigidas a 200 estudiantes de diferentes Carreras.

\section{Resultados}

Actividad 1: Entrevista a los Docentes.

A continuación, se presente un análisis de las encuestas dirigidas a 40 docentes de Institutos Tecnológicos Superiores. La actividad tiene como objetivo, analizar las Tecnologías de la Información y Comunicación (TIC) y su relación en el proceso de enseñanza de la asignatura de Contabilidad, Administración e Informática de diferentes carreras de Educación Superior.

\section{Necesarios las herramientas TIC para el desarrollo de los procesos de enseñanza aprendizaje}

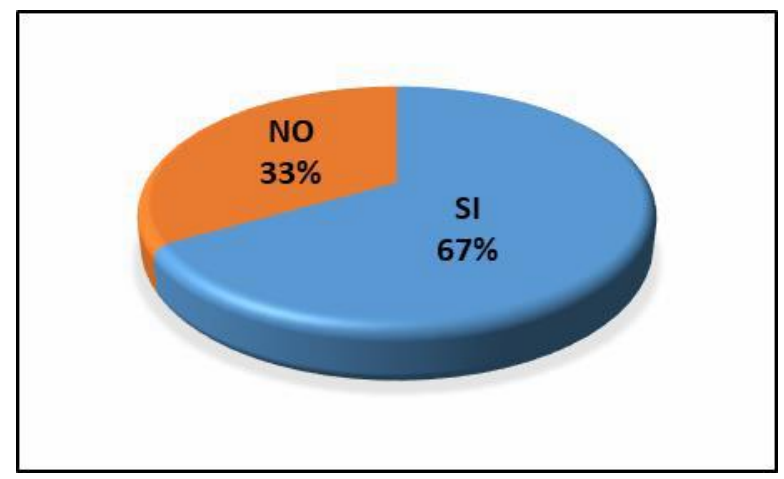

Figura No. 1. Herramientas TIC 
De los resultados de la figura No. 1, se especifica que el $67 \%$ de los encuestados describe que son necesarios las herramientas TIC para el desarrollo de los procesos de enseñanza aprendizaje, mientras que el $33 \%$ especifica que no se necesita.

\section{Uso de equipos tecnológicos en Institutos de educación Superior}

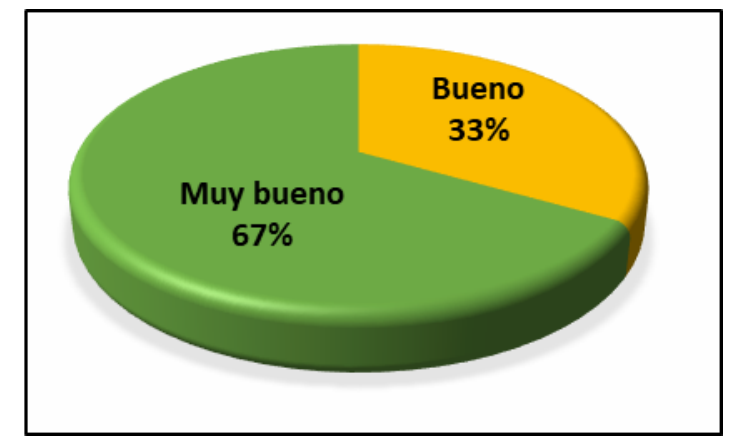

Figura No. 2. Uso de los equipos tecnológicos

En la figura No. 2, el $33.33 \%$ describen que es bueno el uso de la tecnología en el proceso educativo y el $66.67 \%$ especifica que es muy bueno usar las TIC en clase.

\section{Desarrollo de habilidades, competencias, técnicas y estrategias tecnológicas en los docentes}

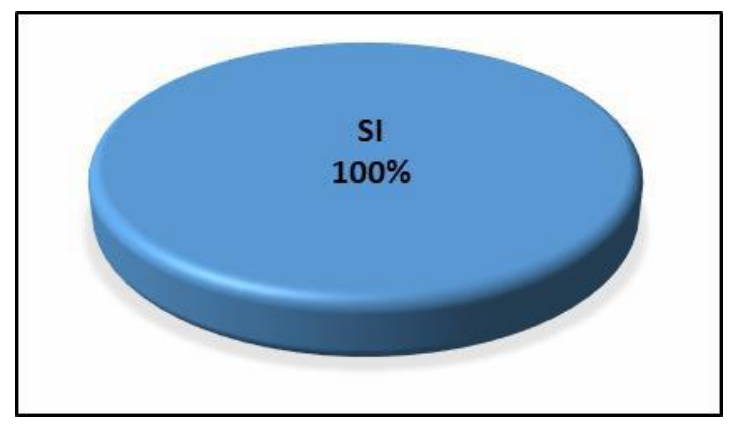

Figura No. 3. Habilidades, competencias, técnicas y estrategias

El $100 \%$ de los docentes encuestados, señala que si es necesario que los profesores desarrollen nuevas competencias, técnicas, habilidades y estrategias basadas en las TIC para lograr un óptimo proceso de enseñanza y una gran concentración de los estudiantes (Ver figura No. 3). 


\section{Las TIC en los procesos educativos en la educación superior}

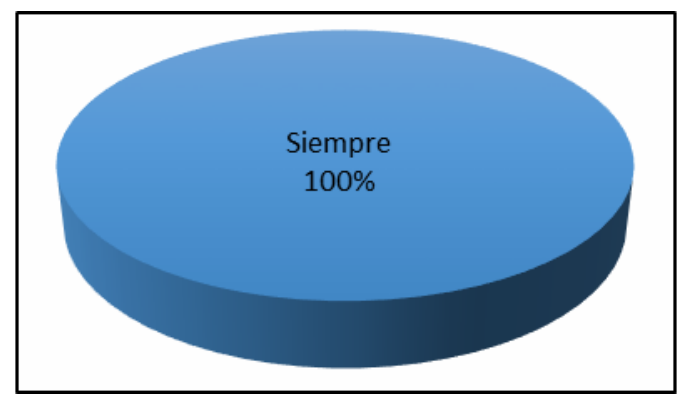

Figura No 1. Uso de las TIC

En figura No. 4, el 100\% describen que siempre es importante el uso de las TIC en los procesos educativos en la educación superior y especialmente en las carreras técnicas.

\section{Utilización de las TIC}

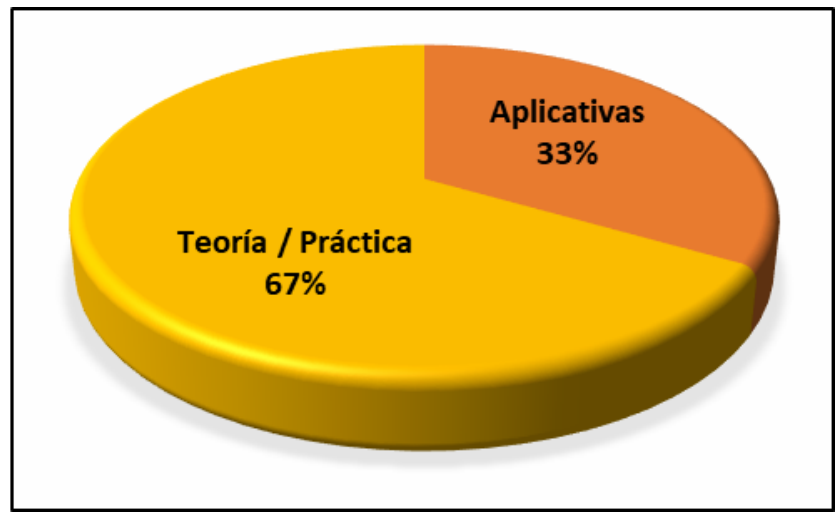

Figura No. 5. Utilizar los docentes las TIC

En dicha pregunta, el 67\% especifican que la mejor forma de impartir la clase es con teoría y práctica, mientras que el $33 \%$ analiza que la forma más óptima es utilizando aplicaciones informáticas (Ver figura No. 5). 


\section{Utilización de las TIC en los Institutos superiores}

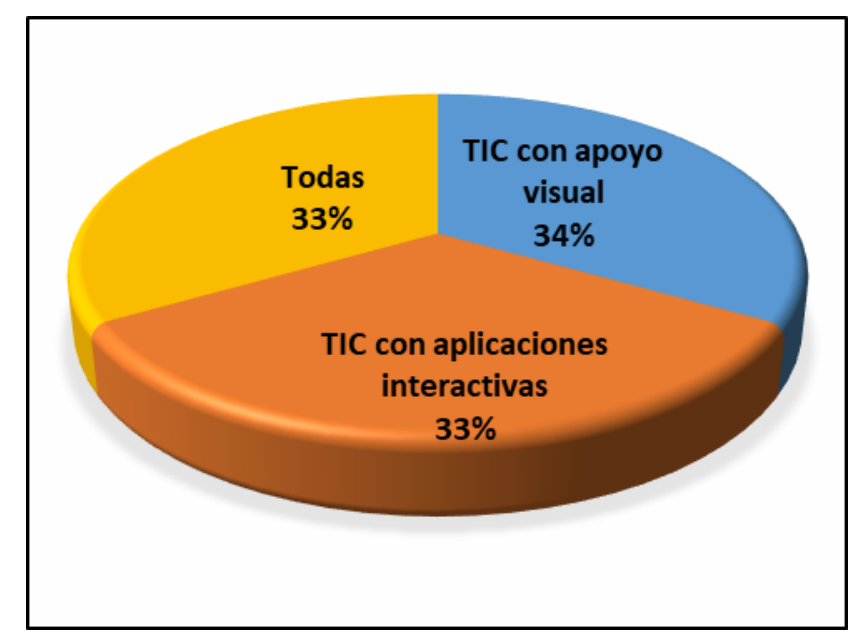

Figura No. 6. Enfoque del uso de las TIC

En la figura No. 6, los docentes encuestados describen que utilizan las TIC con apoyo visual, aplicaciones interactivas y con formatos auditivos en el proceso de enseñanza.

\section{Uso de los recursos didácticos en el aula}

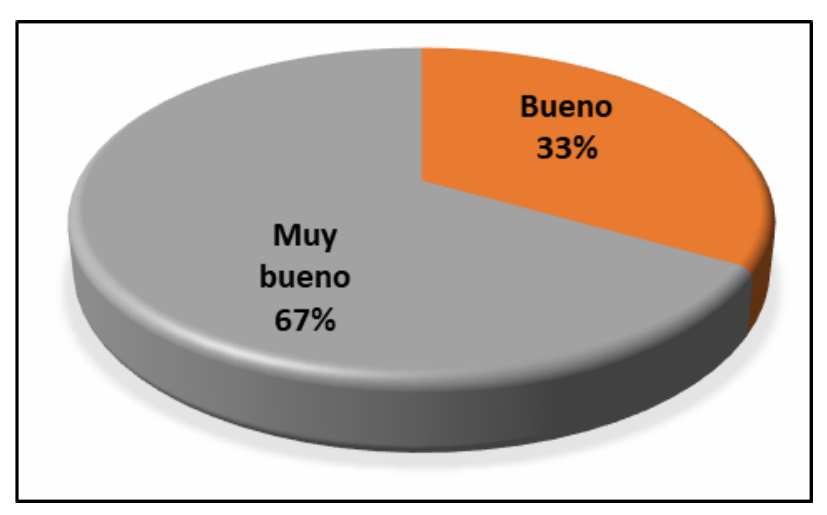

Figura No. 7. Uso de los recursos didácticos en el aula

En la figura No. 7, el 67\% contesta que los recursos didácticos son utilizados de una forma muy buena, y 13 docente (33\%) describe de forma buena. 
Uso de los recursos tecnológicos en el salón de clase

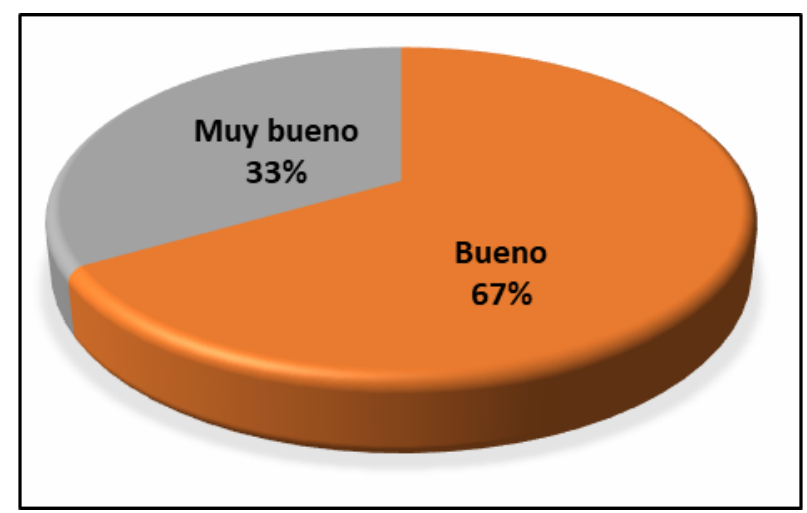

Figura No. 8. Uso de los recursos tecnológicos

En la figura No. 8, 27 docentes encuestados describen que el uso de los recursos tecnológicos en su salón de clases es bueno y 13 de los encuestados describen que su utilización es de forma adecuada y muy buena.

\section{Perdida de la concentración y el interés en clase}

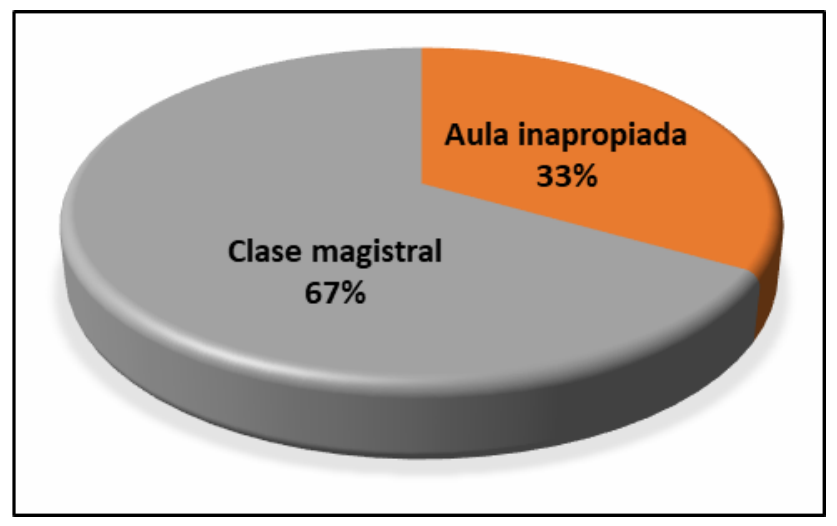

Figura No. 9. Pérdida de la concentración en la clase

En la figura No. 9, los 27 encuestados (67\%) responden que los estudiantes pierden el interés y se desconcentran en clase por que las clases son magistrales, mientras que el $33 \%$ estipula que pueden ser por que el aula es inapropiada. 
Tipos de medios tecnológicos ocupa los docentes en el aula para los procesos de enseñanza aprendizaje

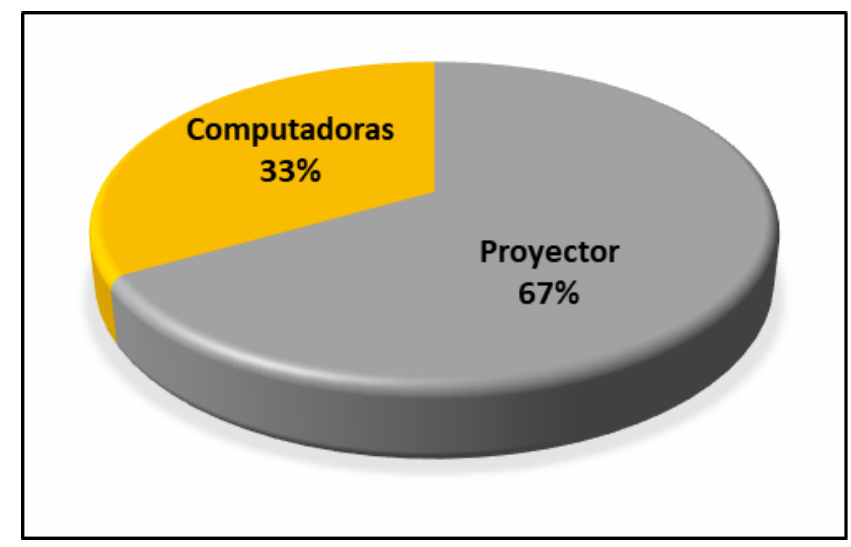

Figura No. 10. Medios tecnológicos

En la figura No. 10, el $67 \%$ especifica que los medios más utilizados para dar la clase son el proyector y un porcentaje minoritario del $33 \%$ describe que son las computadoras.

\section{Actividad 2: Encuestas dirigidas a estudiantes}

A continuación, se presente un análisis de la entrevista dirigido a 200 estudiantes de Institutos Tecnológicos de Educación Superior. El instrumento cuenta con 10 preguntas y el objetivo es Analizar las Tecnologías de la Información y Comunicación (TIC) y su relación en el proceso de enseñanza de las asignaturas de Contabilidad, Administración e Informática en diferentes carreras.

\section{Las herramientas TIC para el desarrollo de los procesos de aprendizaje}

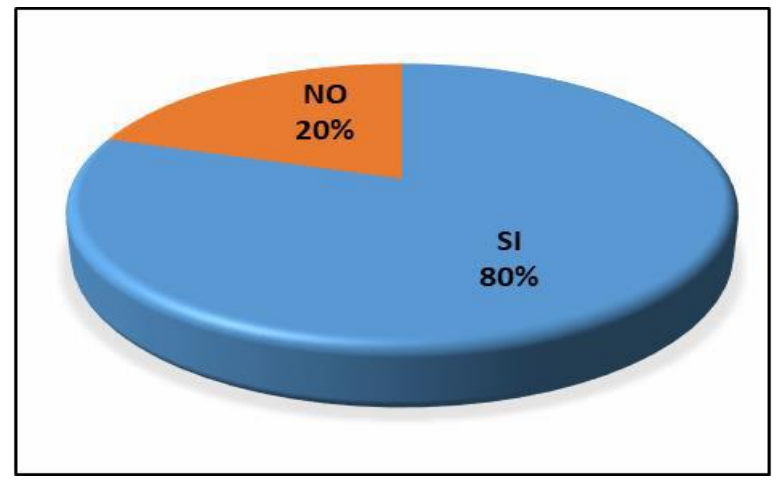

Figura No. 11. Herramientas TIC- Estudiantes 
De los resultados de la figura No. 11, se especifica que, el $80 \%$ de los estudiantes encuestados describe que son necesarios las herramientas TIC en el desarrollo de los procesos de aprendizaje, mientras que el $20 \%$ especifica que no se necesita.

\section{Servicio de los equipos tecnológicos en los laboratorios}

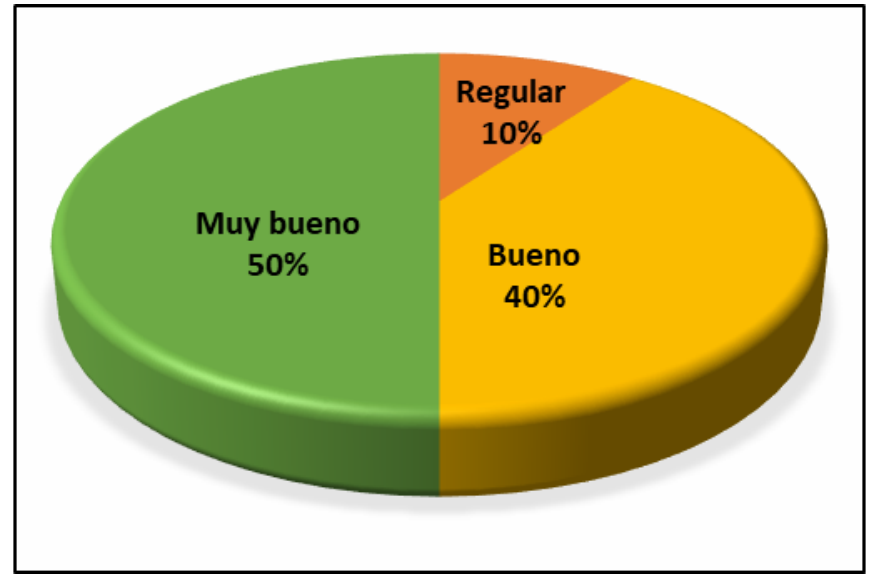

Figura No. 12. Uso de los equipos tecnológicos

En la figura No. 12, el 50\% contesta que es muy bueno el servicio de los equipos tecnológicos que tiene los Institutos, el $40 \%$ describen que es bueno y el 10\% especifica que regular los equipos tecnológicos en los laboratorios.

\section{Nuevas herramientas tecnológicas en los procesos de enseñanza en clase}

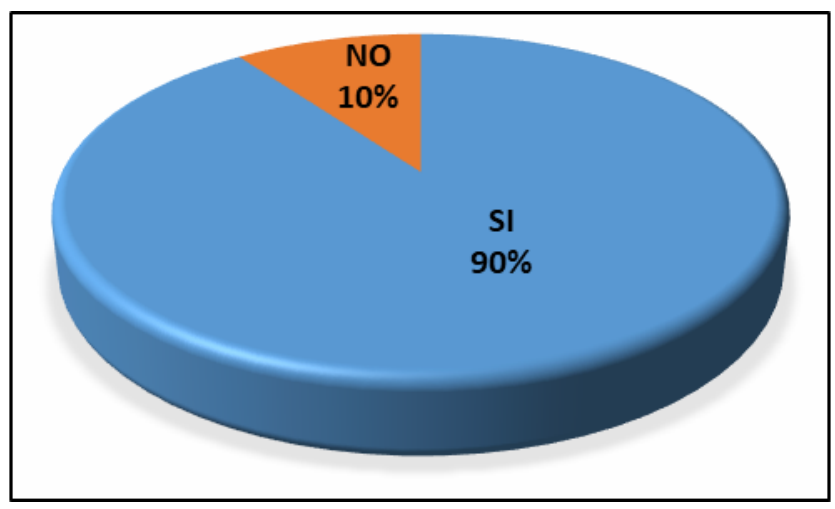

Figura No. 13. Habilidades, competencias, técnicas y estrategias

El $90 \%$ de los estudiantes encuestados señala que, si es necesario que los docentes enseñen con nuevas técnica y herramientas tecnológicas para lograr un óptimo proceso de enseñanza y una 
gran concentración de los estudiantes, mientras que 20 de los estudiantes (10\%) describe que prefiere el modo de enseñanza tradicionalista (Ver figura No. 13).

\section{Uso de las herramientas tecnológicas en la enseñanza de diferentes asignaturas}

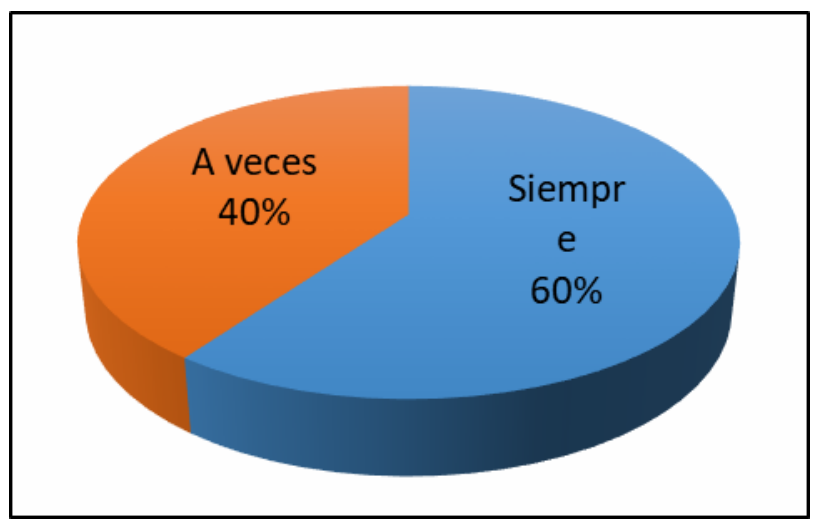

Figura No. 14. Uso de las TIC

En figura No. 14, se puede ver que el $60 \%$ describen que siempre es importante el uso de las TIC y el $40 \%$ dice que a veces es importante el uso de la tecnología en los procesos de enseñanza de las asignaturas, así como también en la educación superior y especialmente en las carreras técnicas.

Tipos de clases para tener una adecuada concentración en la asignatura planteada

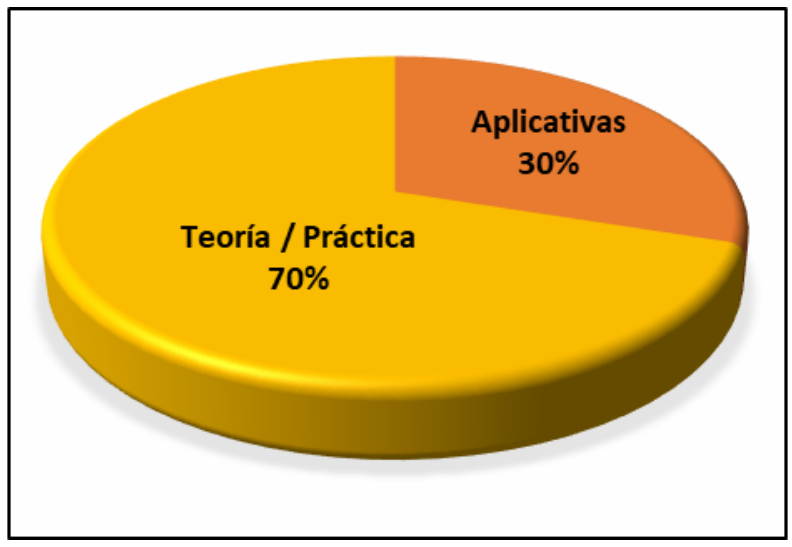

Figura No. 15. Utilizar los docentes las TIC 
En dicha pregunta, 140 estudiantes (70\%) especifican que la mejor forma de impartir la clase es con teoría y práctica, mientras que 60 de los encuestados (30\%) analiza que la forma más óptima es utilizando aplicaciones informáticas (Ver figura No. 15).

\section{Utilización de las TIC en actividades académicos}

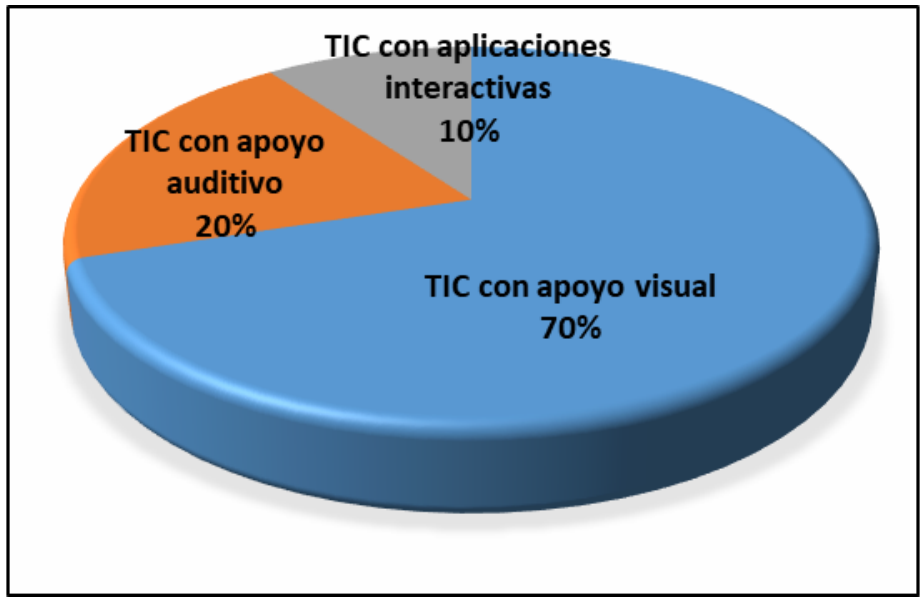

Figura No. 16. Enfoque del uso de las TIC

En la figura No. 16, de los 200 estudiantes encuestados, 140 (70\%) describen que utilizan las TIC en sus actividades académicas con apoyo visual, 40 estudiantes (20\%) dicen que utilizan como apoyo auditivo y el $10 \%$ usa aplicaciones interactivas en el proceso de enseñanza.

\section{Uso de los recursos didácticos en el aula}

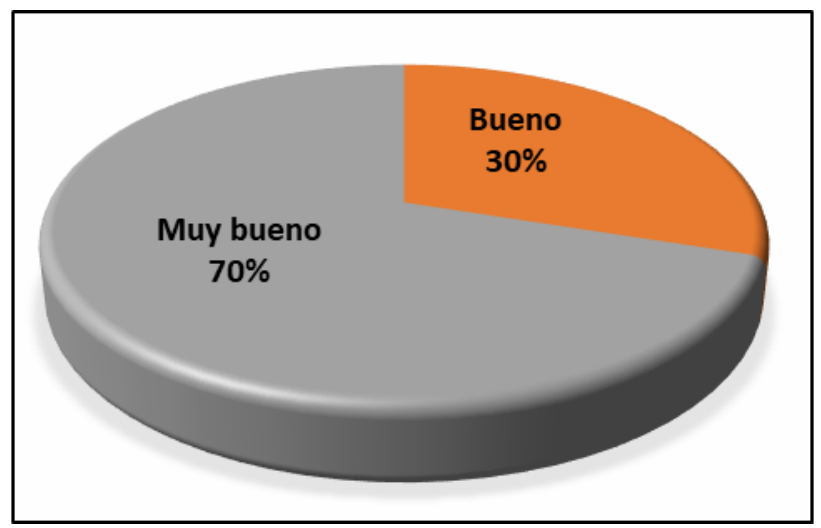

Figura No. 17. Uso de los recursos didácticos en el aula 
En la figura No. 17, el 70\% contesta que los recursos didácticos son utilizados de una forma muy buena, y 60 estudiantes (30\%) describe de forma buena.

\section{Uso de los recursos tecnológicos en el salón de clase}

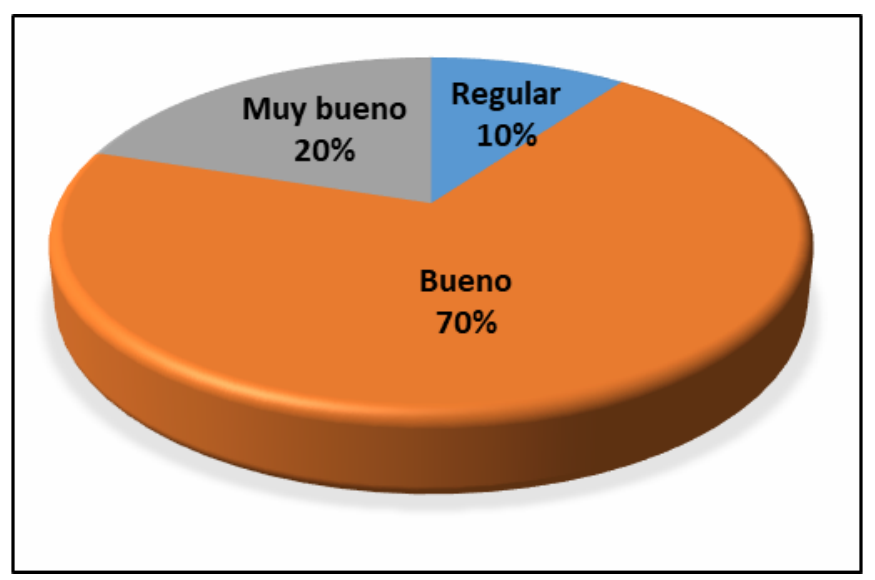

Figura No. 18. Uso de los recursos tecnológicos

En el siguiente punto 40 estudiantes encuestados (20\%) describen que el uso de los recursos tecnológicos en el salón de clases es muy bueno, el 70\% dicen que es buena la utilización de los recursos tecnológicos y 20 de los encuestados (10\%) describen que su utilización es de forma regular (Ver figura No. 18).

\section{Medios tecnológicos utilizados en el aula para los procesos de aprendizaje}

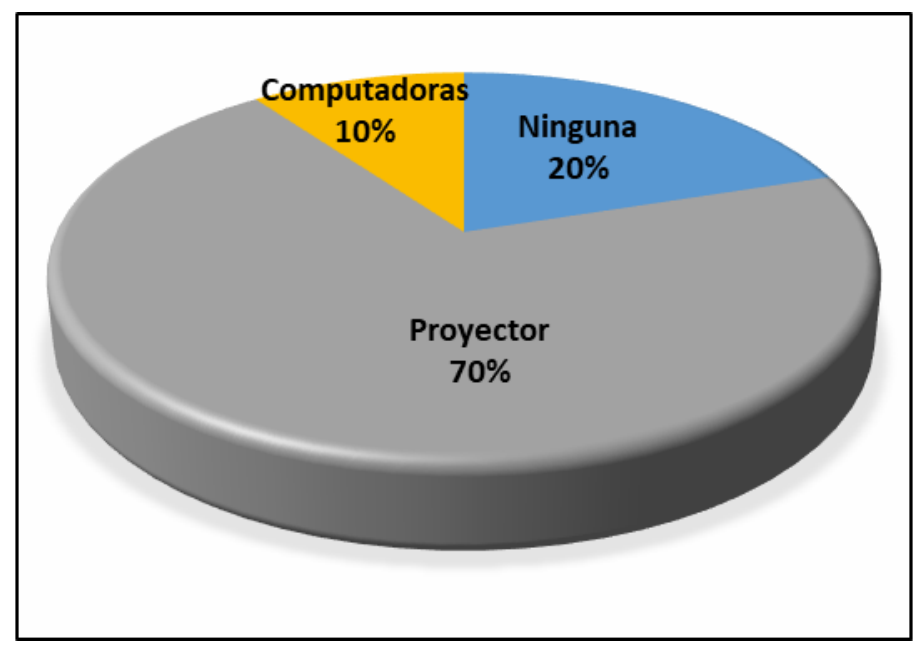

Figura No. 19. Medios tecnológicos 
En la figura No. 19, el $70 \%$ piensa que los medios más utilizados para dar la clase son el proyector, el $10 \%$ describe que son las computadoras y 40 estudiantes informan que no se usa ningún medio tecnológico.

\section{Conclusiones}

Como resultado de la investigación se obtuvo que los procesos de aprendizaje de los estudiantes de la educación superior son más óptimos a través de herramientas tecnológicas, aplicaciones informáticas, procesos educativos online, etc., razón por la cual los nuevos procesos educativos están basado en la educación constructivista y con aprendizaje significativo basado en problemas.

De acuerdo a los antecedentes investigativos, se puede describir que las Tecnologías de la Información y Comunicación han permitido desarrollar nuevas habilidades, estrategias y procesos de enseñanza aprendizaje, logrando así un $60 \%$ más de concentración en los estudiantes universitarios en las diferentes asignaturas.

De acuerdo al Análisis de las preguntas realizadas a los docentes de Institutos Tecnológicos de Educación Superior, se puede especificar que se siguen generando un proceso de enseñanza aprendizaje a través de clases magistrales, utilizando material didáctico en el salón de clase y sin apoyo de las TIC, por lo que más del $60 \%$ de profesores encuestados describen que se necesita las aplicaciones tecnológicas pero no se ha aplicado, también el $100 \%$ de los encuestados afirman que las TIC permiten generar nuevas habilidades, competencias, técnicas y estrategias tecnológicas para una óptima concentración de los estudiantes.

En cuanto al análisis de la encuesta realizada a los estudiantes, el $80 \%$ estipula que se debe utilizar herramientas tecnológicas para un mejor entendimiento de la Asignatura de Contabilidad, Administración e Informática ya que se tratan de materias netamente prácticas, también el 90\% dicen que es necesario que los docentes enseñen las clases con nuevas herramientas tecnológicas ya que esto permitirá incluir la teoría con la práctica y se generará un mundo más real de acuerdo a lo que se vivirá en la vida profesional.

\section{Resumen Bibliográfico}

Burch, S. (2005). Sociedad de la información/Sociedad del conocimiento. Palabras en juego, 5478. 
Uso de las tecnológicas de la información y comunicación (tic) en los institutos de educación superior

Guaña-Moya, E. J.-Q.-R. (2015). Caracterización de entornos virtuales de enseñanza aprendizaje (EVEA) en la educación virtual. Ciencias Holguín.

Guaña-Moya, J. Q.-A.-T. (2018). Estudio preliminar del uso de las redes sociales en los jóvenes ecuatorianos. Revista PUCE, 106.

Moya, E. J. (2017). Utopía o realidad de aplicaciones informáticas en la educación. Caso Universidad Ecuatoriana. Revista Publicando, 119-137.

Salinas, J. (2004). Innovación docente y uso de las TIC en la enseñanza universitaria. International Journal of Educational Technology in Higher Education (ETHE).

Segrera, F. L. (2015). Educación superior comparada: tendencias mundiales y de América Latina y Caribe. Avaliação. Revista da Avaliação da Educação Superior, 21(1).

Valverde Berrocoso, J. (2014). MOOCs: Una visión crítica desde las Ciencias de la Educación. Ministerio de educación. 